\title{
Multi-arm Trial of Inflammatory Signal Inhibitors (MATIS) for Hospitalised Patients with Mild or Moderate COVID-19 Pneumonia: A Structured Summary of a Study Protocol for a Randomised Controlled Trial
}

Nikhil Vergis ( $\nabla$ nvergis@ic.ac.uk)

Imperial College London https://orcid.org/0000-0002-4157-6757

Rachel Phillips

Imperial College London

Victoria Cornelius

Imperial College London

Alexia Katsarou

Imperial College Healthcare NHS Trust

Taryn Youngstein

Imperial College Healthcare NHS Trust

Lucy Cook

Imperial College Healthcare NHS Trust

Michelle Willicombe

Imperial College Healthcare NHS Trust

Clio Pillay

Imperial College Healthcare NHS Trust

Tina Shturova

Imperial College Healthcare NHS Trust

Melanie Almonte

Imperial College Healthcare NHS Trust

Asad Charania

Imperial College Healthcare NHS Trust

Richard Turner

Imperial College Healthcare NHS Trust

Onn Min Kon

Imperial College Healthcare NHS Trust

Graham Cooke

Imperial College London 


\section{Mark Thursz}

Imperial College London

\section{Svetlana Cherlin}

Newcastle University

\section{James Wason}

Newcastle University

\section{Dragana Milojkovic}

Imperial College Healthcare NHS Trust

\section{Andrew Innes}

Imperial College Healthcare NHS Trust

\section{Nichola Cooper}

Imperial College London

\section{Research Article}

Keywords: COVID-19, coronavirus, randomised controlled trial, protocol, pneumonia, fostamatinib, ruxolitinib

Posted Date: March 18th, 2021

DOI: https://doi.org/10.21203/rs.3.rs-322160/v1

License: (c) (i) This work is licensed under a Creative Commons Attribution 4.0 International License. Read Full License

Version of Record: A version of this preprint was published at Trials on April 12th, 2021. See the published version at https://doi.org/10.1186/s13063-021-05190-z. 


\section{Abstract}

The primary objective of MATIS is to determine the efficacy of ruxolitinib (RUX) or fostamatinib (FOS) compared to standard of care (SOC) with respect to reducing the proportion of hospitalised patients progressing from mild or moderate to severe COVID-19 pneumonia.

Secondary objectives, at 14 and 28 days, are to:

Determine the efficacy of RUX or FOS to reduce mortality

Determine the efficacy of RUX or FOS to reduce the need for invasive ventilation or ECMO

Determine the efficacy of RUX or FOS to reduce the need for non-invasive ventilation

Determine the efficacy of RUX or FOS to reduce the proportion of participants suffering significant oxygen desaturation

Determine the efficacy of RUX or FOS to reduce the need for renal replacement therapy

Determine the efficacy of RUX and FOS to reduce the incidence of venous thromboembolism

Determine the efficacy of RUX and FOS to reduce the severity of COVID-19 pneumonia [graded by a 9-point modified WHO Ordinal Scale*

Determine the efficacy of RUX or FOS to reduce systemic inflammation

Determine the efficacy of RUX or FOS to the incidence of renal impairment

Determine the efficacy of RUX or FOS to reduce duration of hospital stay

Evaluate the safety of RUX and FOS for treatment of COVID-19 pneumonia.

\section{Trial design}

A multi-arm, multi-stage (3-arm parallel-group, 2-stage) randomised controlled trial that allocates participants 1:1:1 and tests for superiority in experimental arms versus standard of care.

\section{Participants}

Patients will be recruited while inpatients during hospitalisation for COVID-19 in multiple centres throughout the UK including Imperial College Healthcare NHS Trust.

INCLUSION:

- Patients age $\geq 18$ years at screening 
- Patients with mild or moderate COVID-19 pneumonia, defined as Grade 3 or 4 severity by the WHO COVID-19 Ordinal Scale (Table 1)

- Patients meeting criteria:

- Hospitalization AND

- SARS-CoV2 infection (clinically suspected or laboratory confirmed) AND

- Radiological change consistent with COVID-19 disease

- $\mathrm{CRP} \geq 30 \mathrm{mg} / \mathrm{L}$ at any time point

- Informed consent from patient or personal or professional representative

- Agreement to abstain from sexual intercourse or use contraception that is $>99 \%$ effective for all participants of childbearing potential for 42 days after the last dose of study drug. For male participants, agreement to abstain from sperm donation for 42 days after the last dose of study drug.

\section{EXCLUSION:}

- Requiring either invasive or non-invasive ventilation including CPAP or high flow nasal oxygen at any point after hospital admission but before baseline, not related to a pre-existing condition (e.g., obstructive sleep apnoea)

- Grade $\geq 5$ severity on the modified WHO COVID-19 Ordinal Scale, i.e. $\mathrm{SpO}_{2}<90 \%$ on $\geq 60 \%$ inspired oxygen by facemask at baseline; non-invasive ventilation; or invasive mechanical ventilation

- In the opinion of the investigator, progression to death is inevitable within the next 24 hours, irrespective of the provision of therapy

- Known severe allergic reactions to the investigational agents

- Child-Pugh B or C grade hepatic dysfunction

- Use of drugs within the preceding 14 days that are known to interact with any study treatment (FOS or RUX), as listed in the Summary of Product Characteristics

- Pregnant or breastfeeding

- Any medical condition or concomitant medication that in the opinion of the investigator would compromise subjects' safety or compliance with study procedures.

- Any medical condition which in the opinion of the principal investigator would compromise the scientific integrity of the study

Non-English speakers will be able to join the study. If participants are unable to understand verbal or written information in English, then hospital translation services will be requested at the participating site for the participant where possible.

\section{Intervention and comparator}


RUXOLITINIB (RUX) (14 days): An oral selective and potent inhibitor of Janus Associated Kinases (JAK1 and JAK2) inhibitor and cell proliferation (Verstovek, 2010). It is approved for the treatment of diseaserelated splenomegaly or constitutional symptoms in myelofibrosis, polycythaemia vera and graft-versushost-disease. RUX will be administered orally $10 \mathrm{mg}$ bd Day 1-7 and 5mg bd Day 8-14.

FOSTAMATINIB (FOS) (14 days): An oral spleen tyrosine kinase inhibitor approved for the treatment of thrombocytopenia in adult participants with chronic immune thrombocytopenia. FOS will be administered orally $150 \mathrm{mg}$ bd Day 1-7 and 100mg bd Day 8-14. Please see protocol for recommended dose modifications, where required.

COMPARATOR (Standard of Care, SOC): experimental arms will be compared to participants receiving standard of care. It is accepted that SOC may change during a rapidly evolving pandemic. Co-enrolment to other trials and rescue therapy, either pre- or post-randomisation, is permitted and will be accounted for in the statistical analysis.

\section{Main Outcomes}

Pairwise comparison (RUX vs SOC and FOS vs SOC) of the proportion of participants diagnosed with severe COVID-19 pneumonia within 14 days. Severe COVID-19 pneumonia is defined by a score $\geq 5$ on a modified WHO COVID-19 Ordinal Scale, comprising the following indicators of disease severity:

- Death $O R$

- Requirement for invasive ventilation $O R$

- Requirement for non-invasive ventilation including CPAP or high flow oxygen $O R$

- $\mathrm{O}_{2}$ saturation $<90 \%$ on $\geq 60 \%$ inspired oxygen

\section{Randomisation}

Participants will be allocated to interventions using a central web-based randomisation service that generates random sequences using random permuted blocks (1:1:1), with stratification by age ( $<65$ and $\geq 65$ years) and site.

\section{Blinding (masking)}

No participants or caregivers are blinded to group assignment. Clinical outcomes will be compared blind to group assignment.

\section{Numbers to be randomised (sample size)}

For an early informal dose examination by the Data Monitoring Committee a minimum of 30 participants will be recruited. 
For Stage 1 of this multi-arm multi-stage study, 171 participants will be randomised, with 57 participants in each arm.

If at least one experimental intervention shows promise, then Stage 2 will recruit a further 95 participants per arm. Sample size calculations contained in the protocol.

\section{Trial Status}

Recruitment is ongoing and started $2^{\text {nd }}$ October 2020. We anticipate completion of Stage 1 by July 2021 and Stage 2 by April 2022. The current protocol version 2.0 of $11^{\text {th }}$ February 2021 is appended.

\section{Trial registration}

EudraCT: $2020-001750-22,9^{\text {th }}$ July 2020

ClinicalTrials.gov: NCT04581954, $9^{\text {th }}$ October 2020

\section{Full protocol}

The full protocol is attached as an additional file, accessible from the Trials website (Additional file 1). In the interest of expediting dissemination of this material, the familiar formatting has been eliminated; this Letter serves as a summary of the key elements of the full protocol.

\section{Declarations}

\section{Ethics approval and consent to participate}

The study was approved by the London-Surrey Borders Health Research Ethics Committee 20/HRA/2618, $24^{\text {th }}$ September 2020

We certify that this trial has received ethical approval from the appropriate ethical committee as described above. We will obtain consent from participants to participate in the study or from their personal or professional representative if the patient lacks capacity but might still benefit from inclusion in the study.

\section{Consent for publication}

Not applicable

\section{Availability of data and materials}

Not applicable 
The authors declare that they have no competing interests

\section{Funding}

Funding was provided by Imperial College Biomedical Research Centre, Rigel Pharmaceuticals Ltd and Novartis.

Rigel Pharmaceuticals Ltd and Novartis have played no role in the design of the study; in the collection, analysis and interpretation of data; nor in the writing of the manuscript.

\section{Authors' contributions}

$N V$ developed the protocol and wrote the manuscript, $A J I, T Y, D M, L C, M W, C P, T S, M A, A C, R T, O M K, S C, R P$, $J W, V C, A K, G C, M R T$ developed protocol and revised the manuscript. NC is Chief Investigator of the study; secured funding; developed the protocol and revised the manuscript. All authors read and approved the final manuscript.

\section{Acknowledgements}

We acknowledge support from the Imperial College Biomedical Research Centre, London.

\section{Supplementary Files}

This is a list of supplementary files associated with this preprint. Click to download.

- MATISProtocolv2.011Feb21SA3CLEAN.pdf 